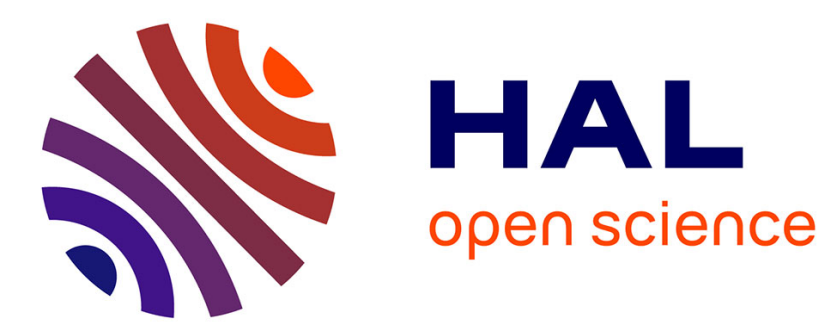

\title{
Non-negative matrix factorization for single-channel EEG artifact rejection
}

\author{
Cécilia Damon, Antoine Liutkus, Alexandre Gramfort, Slim Essid
}

\section{To cite this version:}

Cécilia Damon, Antoine Liutkus, Alexandre Gramfort, Slim Essid. Non-negative matrix factorization for single-channel EEG artifact rejection. ICASSP, 2013, Vancouver, Canada. 10.1109/ICASSP.2013.6637836 . hal-00958775

\section{HAL Id: hal-00958775}

\section{https://hal.inria.fr/hal-00958775}

Submitted on 13 Mar 2014

HAL is a multi-disciplinary open access archive for the deposit and dissemination of scientific research documents, whether they are published or not. The documents may come from teaching and research institutions in France or abroad, or from public or private research centers.
L'archive ouverte pluridisciplinaire HAL, est destinée au dépôt et à la diffusion de documents scientifiques de niveau recherche, publiés ou non, émanant des établissements d'enseignement et de recherche français ou étrangers, des laboratoires publics ou privés. 


\title{
NON-NEGATIVE MATRIX FACTORIZATION FOR SINGLE-CHANNEL EEG ARTIFACT REJECTION
}

\author{
Cécilia Damon $\quad$ Antoine Liutkus ${ }^{\star} \quad$ Alexandre Gramfort ${ }^{\star} \quad$ Slim Essid ${ }^{\star}$ \\ * Institut Mines-Telecom, TELECOM ParisTech - CNRS, LTCI \\ 37, rue Dareau 75014 Paris, France
}

\begin{abstract}
New applications of Electroencephalographic recording (EEG) pose new challenges in terms of artifact removal. In our work we target applications where the EEG is to be captured by a single electrode and a number of additional lightweight sensors are allowed. Thus, this paper introduces a new method for artifact removal for single-channel EEG recordings using nonnegative matrix factorisation (NMF) in a Gaussian source separation framework. We focus the study on ocular artifacts and show that by properly exploiting prior information on the latter, through the analysis of electrooculographic recordings, our artifact removal results on single-channel EEG are comparable to the results obtained with the classic multi-channel Independent Component Analysis technique.
\end{abstract}

Index Terms - EEG, artifact removal, nonnegative matrix factorisation, source separation, Gaussian model.

\section{INTRODUCTION}

Electroencephalographic (EEG) recordings capture a mixture of endogenous brain activities and extraneous environmental and physiological artifacts such as power grid noise, eye movements, heart beat or muscle activities. These artifacts make it difficult to analyze and interpret EEG data since they tend to overlap with the cerebral signals of interest, which are generally of lower energy than the artifacts [15]. Consequently researchers (in the neuroscience and biomedical engineering communities) have devised EEG artifact removal techniques that have proven effective for particular sources of artifacts such as eye movements or heart beats $[4,11]$. However many such techniques can be considered as being mainly geared towards experimental (especially medical) setups. Indeed, the sources of artifacts are generally intentionally limited, especially by requiring that the subject wearing the EEG device remain as steady as possible, and the processing occurs offline, possibly requiring a user's intervention to complete the artifact decontamination process. The latter is in particular true for one of the most popular approaches which relies on Independent Component Analysis (ICA) [12].

Nevertheless new applications of EEG recording, for instance brain-computer interfaces or human-activity monitor- ing, pose new challenges in terms of artifact removal as they call for fully automatic techniques, that would be additionally amenable to real-time processing. A few proposals have been made along this line in previous works where two types of methods can be distinguished. The first exploit various statistical properties of the artifacts in the time- and frequencydomains [14, 18], the second rely on prior knowledge about either the artifacts $[9,19]$ or the signal of interest [21]. The former are specific to standard artifacts whereas the latter can integrate different kinds of prior information.

A common major drawback to such techniques, which is actually common to all ICA-based approaches, is the fact that they can only handle overdetermined mixtures, which entails using many EEG electrodes (at least as many as the sources of artifacts plus one for the useful information). This is clearly prohibitive for the general public applications that we are interested in, where the EEG setup is to be maintained as light as possible and ideally be limited to a single electrode, while allowing the use of other types of lightweight sensors, for example Electromyographic (EMG), Electrocardiographic (ECG), or inertial measurement sensors.

Therefore attempts at single-channel EEG artifact removals have been made [10]. Our work falls in this category.

We propose a novel automatic artifact removal scheme, inspired by the current state-of-the-art in underdetermined audio source separation $[20,7,16]$, exploiting nonnegative matrix/tensor factorization (NMF) in a probabilistic Gaussian framework.

While NMF has already been used for EEG-feature extraction [13], its use for EEG artifact removal within a Gaussian source separation framework is to our knowledge completely novel. Our method is further described in Section 2 before an experimental validation is proposed in Section 3 where our approach is compared to the classic ICA method on real EEG recordings. While these experiments focus on artifacts related to eye movements and blinks (exploiting Electrooculographic (EOG) recordings) being a strong source of contamination, it is important to note that the advantage of our method is its potential to handle many concurrent sources of artifacts simultaneously even when a single EEG electrode is used for recording, provided that some auxiliary signals describing the temporal activations of those sources of artifacts 
can be captured, which is often easily realized.

It is worth mentioning that our approach is advantageous compared to regression techniques used in numerous works on EEG artifact rejection [8] which required the use of "clean" recordings of sources of artifacts. In particular when treating ocular artifacts, the EOG recordings used to estimate the artifact signals tend to be contaminated by the EEG signals which are then subtracted from the brain signals of interest, hence causing a serious difficulty for such regression techniques.

\section{NMF-BASED ARTIFCAT REJECTION}

We hereafter explain how nonnegative matrix factorisation is used in order to perform EEG artifact rejection following a probabilistic blind source separation paradigm. We first expose the theoretical foundations and the general model underlying the separation procedure adopted, then we describe how the latter is specifically applied to our particular data configuration, that is single-channel EEG accompanied with auxiliary signals describing the sources of artifacts.

\subsection{Probabilistic model and general separation proce- dure}

For the sake of generality, we suppose that there are $I$ observable time-series $\tilde{x}(t, i)$, each $\tilde{x}(\cdot, i)$ corresponding to one of the EEG sensors. For a given sensor $i$, we assume that each $\tilde{x}(\cdot, i)$ is the sum of $J$ underlying signals $\tilde{y}(\cdot, i, 1), \ldots, \tilde{y}(\cdot, i, J)$ which are called latent components in this study. Hence, we simply have :

$$
\forall(t, i), \tilde{x}(t, i)=\sum_{j=1}^{J} \tilde{y}(t, i, j) .
$$

Thus, model (1) can be understood as stating that the observed $I$ time-series can actually be decomposed as the sum of $J$ latent sets of $I$ time-series. The objective of the processing under study then becomes to extract those $J$ sets. That way, each $\tilde{x}(\cdot, i)$ of the observed EEG data will be decomposed into its $J$ constituent $\{\tilde{y}(\cdot, i, j)\}_{j=1, \ldots, J}$ latent components. In this paper, a distinction is made between stochastic processes such as $\tilde{y}(\cdot, i, j)$ or $\tilde{x}(\cdot, i)$ and their realizations $\tilde{\mathbf{y}}(\cdot, i, j)$ and $\tilde{\mathbf{x}}(\cdot, i)$ which are written in bold type.

In the following, we will make use of a Time-Frequency (TF) representation of the signals considered. More specifically, $x(\cdot, \cdot, i)$ will denote the Short Term Fourier Transform (STFT) of the mixture $\tilde{x}(\cdot, i)$, so that $x(f, n, i) \in \mathbb{C}$ is its spectrum at frequency bin $f$ for frame index $n$. Similarly, $y(f, n, i, j)$ denotes the STFT of the $i^{\text {th }}$ channel of latent component $j$ at TF bin $(f, n)$. All signals are supposed to have the same number $F$ of frequency indices and the same number $N$ of frames.
Following [16], all $\{\tilde{y}(\cdot, i, j)\}_{i, j}$ are here supposed to be independent and Locally Stationary Gaussian Processes (LSGP, see [16]). The main attractive feature of this model is that computations can be readily performed in the STFT representation. Indeed it can be be shown $[1,2,16]$ that Minimum Mean Squared Error (MMSE) estimates of the latent components are then given through WIENER filtering by :

$$
\hat{\mathbf{y}}(f, n, i, j)=\frac{P(f, n, i, j)}{\sum_{j=1}^{J} P(f, n, i, j)} \mathbf{x}(f, n, i)
$$

where $P(f, n, i, j)>0$ is the Power Spectral Density (PSD) of the $i^{\text {th }}$ channel of component $j$ at TF bin $(f, n)$.

This separation procedure is very simple to implement. One just has to compute the STFTs of the observed EEG signals $\tilde{\mathbf{x}}(\cdot, i)$, and then compute (2) for all $(f, n, i, j)$. Separated time series are then simply recovered through an inverse STFT procedure, which consists in inverse Fourier transforms followed by overlap-add procedures.

The main issue with the proposed model is of course the important number $F N I J$ of its parameters $P(f, n, i, j)$. Still, one of its most interesting features is the way prior knowledge can be taken into account in order to strongly reduce the number of its parameters. Indeed, several facts can be considered for modeling $P$ :

- Even if the $I$ different channels of each latent component are modeled as independent, their Power Spectral Densities may be strongly related. In this study, we will assume that :

$$
P(f, n, i, j)=Q_{i j} P(f, n, j),
$$

with $Q_{i j} \geq 0$. Equation (3) amounts to writing that all channels of a given component $j$ share the same PSD $P(f, n, j)$ up to a nonnegative scaling factor $Q_{i j}$.

- For one given latent component $j$, we may assume that its PSD $P(f, n, j)$ as it appears in (3) is further structured so that it simply consists of one given spectral template $W_{j}$ that is modulated by a time-varying activation gain $H_{j}$. This can be written :

$$
P(f, n, j)=\left[W_{j} H_{j}^{\top}\right]_{f, n},
$$

where $W_{j}$ and $H_{j}$ are $F \times 1$ and $N \times 1$ nonnegative vectors.

As can be seen, these two simplifications lead to a reduction of the number of parameters from FNIJ to $(F+N+I) J$, which is quite remarkable. We are then left with the problem of modeling the PSD of observed signals $\tilde{x}(\cdot, i)$ using a Nonnegative Tensor Factorization (NTF [3]), which simplifies to Nonnegative Matrix Factorisation (NMF) in the single-channel case. Hence, though we here focus on the latter situation, the method that we present can be also used for multi-sensor data. 


\subsection{NMF decomposition}

A fact which is now acknowledged in the litterature [5, 16] is that learning a NMF model such as (4) through maximum likelihood estimation is equivalent to minimizing the ItakuraSaito (IS) divergence ${ }^{1}$ between the power spectrogram of the observations and the model :

$$
\begin{array}{r}
\{\hat{W}, \hat{H}, \hat{Q}\}= \\
\underset{W, H, Q}{\operatorname{argmin}} \sum_{f, n, i} d_{I S}\left(|\mathbf{x}(f, n, i)|^{2} \| \sum_{j=1}^{J} W_{f j} H_{n j} Q_{i j}\right),
\end{array}
$$

where $W_{j}$ and $H_{j}$ have been gathered as the $J$ columns of matrices $W$ and $H$ of respective dimensions $F \times J$ and $N \times J$. Very efficient iterative algorithms exist which permit to estimate $W, H$ and $Q$ that minimize (5). The reader is referred to studies such as $[6,17,20]$ for the details of those algorithms. The NMF problem may also be solved using other cost functions such as the Euclidean distance (i.e. $l_{2}$ norm) and the Kullback-Leibler divergence commonly used in the NMF litterature [5]. In our experiments (presented in Section 3) the use of the Itakura-Saito cost has been assessed by comparison to results obtained based on Euclidean cost functions.

The main issue with this approach lies in the fact that the latent components obtained that way, even if they correctly sum up to the observed mixtures, do not necessarily correspond to the latent components we are looking for. To address this issue, we propose an approach based on supervised initialization of the model parameters.

\subsection{Informed NMF initialization}

One important feature of the current work is the use of auxiliary signals characterizing the sources of artifacts to aid their rejection in single-channel EEG analysis. This is achieved by initializing the learning process with results of the NMF decomposition of such auxiliary signals.

In contrast, single-channel source separation is not possible with ICA which is constrained to be used in (over)determined settings. Still, ICA can equally benefit from the initialization of components to be extracted with the available auxiliary signals, which is actually what we do when we compare our method to ICA. However, the latter requires the addition of a new EEG channel for each new source of artifact so that the minimum number of EEG channels that need to be used to handle $L$ sources of artifacts must be $L+1$ (one channel per artifact component plus one for EEG useful information). Using NMF source separation, this restriction is removed and we are able to integrate multiple known artifact sources in the learning process with a single EEG channel.

\footnotetext{
${ }^{1}$ The Itakura-Saito divergence between two nonnegative scalars $a$ and $b$ is defined as $d_{I S}(a \mid b)=\frac{a}{b}-\log \frac{a}{b}-1$.
}

We proceed as follows. As a first step, we perform a NMF decomposition on each reference source providing $K^{i n i t}$ spectral components $W^{i n i t}$ and activation gains $H^{i n i t}$. Then we use these activations, $H^{i n i t}$, to initialize the NMF decomposition of the EEG data with $K$ components such that $K>K^{i n i t}$. This strategy has proven more effective than the alternative consisting in using $W^{i n i t}$ instead, as it better copes with the variability of the spectral patterns characterizing the artifacts (captured in $W^{i n i t}$ ) across different sensors. The remaining $K-K^{\text {init }}$ components of the EEG NMF decomposition are randomly initialized. Once this NMF model is learned, the artifact and decontaminated EEG signals can be easily reconstructed through WIENER filtering.

\section{EXPERIMENTS AND RESULTS}

We now present results on real EEG signals corrupted by ocular artifacts. We mainly aim at proving the efficiency of NMF in removing ocular artifact in single channel EEG analysis comparing to 2 channel-based ICA source separation.

\subsection{Validation procedure}

Constrained FastICA and NMF source separation methods are here used with a given EOG reference channel to automatically remove ocular artifacts. The EEG data are public ${ }^{2}$ and were acquired at Martinos Center for Biomedical Imaging on a single subject with a 60 EEG-electrode cap combined with a 306-channel MEG Neuromag Vectorview system in a magnetically shielded room. Simultaneously, ocular movements were recorded with a vertical EOG channel. The experiment consists of audio and visual stimuli. The data was recorded at a rate of $600 \mathrm{~Hz}$. We worked on 2 particular EEG channels located to the front of the cap being highly contaminated by eye movements.

As described in Section 2.3, for both methods initialization is performed with the given artifact reference signal to guide the source learning process. For FastICA, this merely consists in initializing a component of the mixing matrix with the EOG signal and the other components are generated randomly. For NMF, the initialization is done as described in 2.3.

The validation procedure includes two steps : a training step during which the hyperparameters of each source separation method are learned on one half of the dataset and a test step during which the best hyperparameter is tested on the other half of the dataset. Both centered EEG and EOG data are split in two sets to perform the initialization. Five models with 100 iterations each have been learned on both EEG- and EOG-based NMF models and only those with the smallest cost-function value have been selected.

While we necessarily estimated only 2 components for ICA, we have been able to test a range of hyperparameters for

\footnotetext{
${ }^{2}$ http://martinos.org/mne/
} 
NMF by varying the number of components assessed to the artifact on the one hand (i.e. 2,4,6,8), and to EEG sources on the other hand (i.e. $4,6,8,16$ ).

To compare ICA and NMF results, we used two similarity measures, the mutual information and the correlation, and defined two criteria. The first criterion assesses the similarity between the estimated artifact and EOG signals while the second assesses the EEG denoising quality by computing the ratio of the similarity measure between the noise-suppressed and EOG signal to the similarity measure between the original and EOG signals. Below, we present the results of this comparison.

\subsection{Results}

We only show the NMF results obtained with the ItakuraSaito cost function (that corresponds to maximum likelihood inference in our Gaussian framework). Our tests with NMF using the $l_{2}$ norm have completely failed. This can be explained by a nice feature of the Itakura-Saito cost, that is its scale invariance, which makes it more robust to the varying dynamics of EEG and EOG signals.

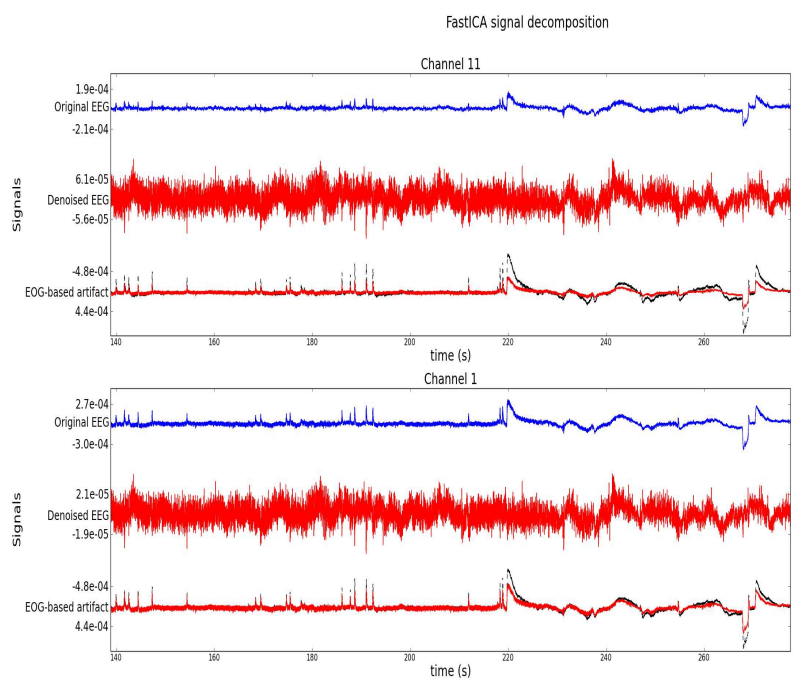

Fig. 1. FastICA signal decomposition on two frontal EEG channels. In order of appearance, the blue signal is the original signal, the red signal is the denoised EEG signal and the last signals include the EOG signal in black dotted line and the estimated artifact signal in red solid line.

Both methods have succeeded in extracting the eye movement artifact signal and thus in denoising the EEG signal. The bar graph and the signals visual inspection prove that the NMF approach with one channel is as efficient as the FastICA method with two channels.

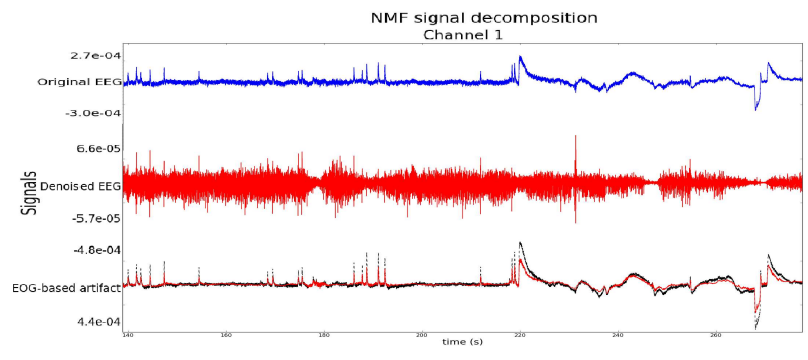

Fig. 2. NMF signal decomposition on a single EEG channel with eight EEG components and four artifact components. In order of appearance, the blue signal is the original signal, the red signal is the denoised signal and the last signals include the EOG signal in black dotted line and the estimated artifact signal in red solid line.

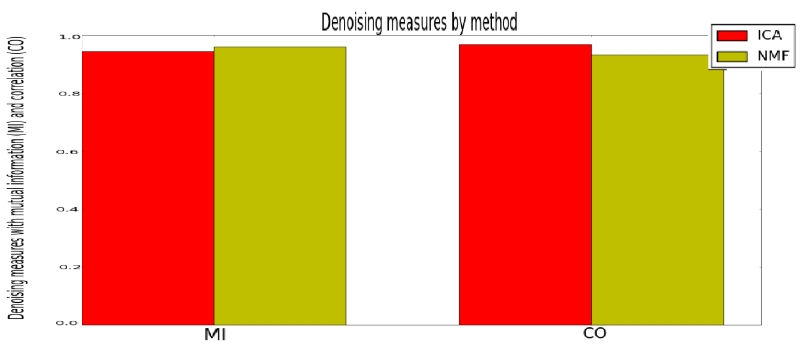

Fig. 3. Mutual information-based and correlation-based denoising measures for each method, ICA in red bars and NMF in green bars. Denoising measures are averaged over channels for ICA.

\section{CONCLUSION}

In this study we showed how NMF can address the problem of EEG artifact removal even in underdetermined settings, namely when the number of EEG channels is less than the number of brain sources and noisy signals (heart beat, eye blinks, etc.). Our experimental results on real data are promising for modern applications of EEG with lightweight devices more and more widely available on the market.

Future work will consider many concurrent sources of artifacts including less-studied ones occurring when the subjects are in motion.

\section{ACKNOWLEDGMENT}

This work was supported by the DGA and the DGCIS under the MEEGAPERF project. 


\section{REFERENCES}

[1] L. Benaroya, F. Bimbot, and R. Gribonval. Audio source separation with a single sensor. IEEE Trans. on Audio, Speech and Language Processing, 14(1):191-199, January 2006.

[2] A.T. Cemgil, P. Peeling, O. Dikmen, and S. Godsill. Prior structures for Time-Frequency energy distributions. In Proc. of the 2007 IEEE Workshop on. App. of Signal Proc. to Audio and Acoust. (WASPAA), pages 151-154, NY, USA, October 2007.

[3] A. Cichocki, R. Zdunek, A. H. Phan, and S. Amari. Nonnegative Matrix and Tensor Factorizations: Applications to Exploratory Multi-way Data Analysis and Blind Source Separation. Wiley Publishing, September 2009.

[4] R.J. Croft and RJ Barry. Removal of ocular artifact from the EEG: a review. Neurophysiologie Clinique/Clinical Neurophysiology, 30(1):5-19, 2000.

[5] C. Févotte, N. Bertin, and J.-L. Durrieu. Nonnegative matrix factorization with the Itakura-Saito divergence. With application to music analysis. Neural Computation, 21(3):793-830, March 2009.

[6] C. Févotte and J. Idier. Algorithms for nonnegative matrix factorization with the beta-divergence. Neural Computation, 23(9):2421-2456, Sep. 2011.

[7] C. Févotte and A. Ozerov. Notes on nonnegative tensor factorization of the spectrogram for audio source separation : statistical insights and towards self-clustering of the spatial cues. In 7th International Symposium on Computer Music Modeling and Retrieval (CMMR 2010), 2010.

[8] G Gratton, M G Coles, and E Donchin. A new method for off-line removal of ocular artifact. Electroencephalography and clinical neurophysiology, 55(4):468-84, April 1983.

[9] C. J. James and O. J. Gibson. Temporally constrained ICA: an application to artifact rejection in electromagnetic brain signal analysis. IEEE Trans Biomed Eng, 50(9):1108-1116, Sep 2003.

[10] C. J. James and D. Lowe. Extracting multisource brain activity from a single electromagnetic channel. Artif Intell Med, 28(1):89-104, May 2003.

[11] T-p P. Jung, S. Makeig, C. Humphries, T-w W. Lee, M. J. Mckeown, V. Iragui, T. J. Sejnowski, and S A N Diego. Removing electroencephalographic artifacts by blind source separation. Psychophysiology, 37(2):163$178,2000$.
[12] T.P. Jung, C. Humphries, T.W. Lee, S. Makeig, M.J. McKeown, V. Iragui, T.J. Sejnowski, et al. Extended ica removes artifacts from electroencephalographic recordings. Advances in neural information processing systems, pages 894-900, 1998.

[13] H. Lee and S. Choi. Group nonnegative matrix factorization for EEG classification. In Proceedings of the International Conference on Artificial Intelligence and Statistics (AISTATS), Clearwater Beach, Florida, 2009.

[14] P. LeVan, E. Urrestarazu, and J. Gotman. A system for automatic artifact removal in ictal scalp EEG based on independent component analysis and Bayesian classification. Clin Neurophysiol, 117(4):912-927, Apr 2006.

[15] O G Lins, T W Picton, P Berg, and M Scherg. Ocular artifacts in EEG and event-related potentials I: Scalp topography. Brain topography, 6(1):51-63, January 1993.

[16] A. Liutkus, R. Badeau, and G. Richard. Gaussian processes for underdetermined source separation. IEEE Transactions on Signal Processing, 59(7):3155 -3167, July 2011.

[17] A. Liutkus, J. Pinel, R. Badeau, L. Girin, and G. Richard. Informed source separation through spectrogram coding and data embedding. Signal Processing, 92(8):1937 - 1949, 2012.

[18] N. Mammone, F. La Foresta, and F.C. Morabito. Automatic artifact rejection from multichannel scalp EEG by wavelet ica. Sensors Journal, IEEE, 12(3):533 -542, march 2012.

[19] M.K.I. Molla, T. Tanaka, and T.M. Rutkowski. Multivariate emd based approach to eog artifacts separation from EEG. In Acoustics, Speech and Signal Processing (ICASSP), 2012 IEEE International Conference on, pages 653-656, march 2012.

[20] A. Ozerov, E. Vincent, and F. Bimbot. A general flexible framework for the handling of prior information in audio source separation. Audio, Speech, and Language Processing, IEEE Transactions on, PP(99):1, 2011.

[21] Yuan Zou, J. Hart, and R. Jafari. Automatic EEG artifact removal based on ica and hierarchical clustering. In Acoustics, Speech and Signal Processing (ICASSP), 2012 IEEE International Conference on, pages 649 652, march 2012. 